\title{
A REMARK ON ANGULAR COMPLEX DILATATIONS OF QUASICONFORMAL MAPPINGS
}

\author{
RICHARD FEHLMANN
}

(Communicated by Irwin Kra)

\begin{abstract}
By a theorem of Ortel an angular complex dilatation $\kappa$ is extremal iff it is Teichmüller (quadratic differential with finite norm) or if it satisfies an integral condition involving the angular limits $\lambda_{x}(\vartheta)$. We show that this second case occurs iff $\lambda_{x}(\vartheta)$ can be given explicitly at a certain point $x$, namely by $\lambda_{x}(\vartheta)=\|\kappa\|_{\infty} e^{2 i\left(\vartheta-\vartheta_{0}\right)}$. Moreover, we investigate this statement under the weaker condition of angularity when the uniformity part in its definition is dropped.
\end{abstract}

1. Introduction. Let $\kappa$ be a measurable complex-valued function in the upper half-plane $H$ with $\|\kappa\|_{\infty}=\operatorname{ess}_{\sup _{z \in H}}|\kappa(z)|<1$. Then $\kappa$ is the complex dilatation of a qc (quasiconformal) self-mapping $f$ of $H$ onto itself, i.e., $\kappa=f_{\bar{z}} / f_{z}$ and $f$ is uniquely determined if we prescribe e.g. $f(0)=0, f(1)=1$ and $f(\infty)=\infty$. We call $\kappa$ extremal, if $\|\kappa\|_{\infty}$ is minimal among all complex dilatations of qc self-mappings of $H$ with the same boundary values as $f$. Then extremality occurs if and only if the Hamilton condition holds, i.e.,

$$
\sup _{\substack{\varphi \in B(H) \\\|\varphi\|=1}}\left|\iint \kappa(z) \varphi(z) d x d y\right|=\|\kappa\|_{\infty}
$$

(see [H and R-St]). Here, $B(H)$ denotes the Banach space of analytic functions in $H$ with finite $L_{1}$-norm $\|\varphi\|=\int_{H} \int|\varphi(z)| d x d y$. Well-known examples of extremal $\kappa$ 's are complex dilatations of Teichmüller mappings with associated quadratic differentials of finite $L_{1}$-norm (see [St1 and R-St]) in which case we call $\kappa$ T.f.n.

If such a $L_{\infty}$-function $\kappa$ is given explicitly, e.g. a piecewise constant function, it can be difficult to check this condition (1.1) directly. For this purpose, M. Ortel has investigated angular complex dilatations [02]. We call $\kappa$ angular at the boundary point $x \in \mathbf{R}$ if there exists a measurable function $\lambda_{x}(\vartheta)$ such that

$$
\kappa\left(x+r e^{i \vartheta}\right) \stackrel{r \downarrow 0}{\longrightarrow} \lambda_{x}(\vartheta) \text { for a.e. } \vartheta \in(0, \pi),
$$

and we call $\kappa$ u.a.b.-angular at the point $x \in \mathbf{R}$ (uniformly at the boundary) if, additionally, the limit (1.2) holds uniformly for $\vartheta \in\left(0, \varepsilon_{x}\right) \cup\left(\pi-\varepsilon_{x}, \pi\right)$ for some $\varepsilon_{x}>0 . \kappa$ is called angular or u.a.b.-angular if it has this property at every boundary point $x \in \overline{\mathbf{R}}=\mathbf{R} \cup\{\infty\}$. For $x=\infty$ these definitions are adjusted in the obvious way. M. Ortel then derives the following theorem from the Hamilton condition:

Received by the editors December 9, 1987.

1980 Mathematics Subject Classification (1985 Revision). Primary 30 C60.

The author is supported by the Schweizerischer Nationalfonds zur Förderung der wissenschaftlichen Forschung. 
THEOREM 1.1 [02]. Let $\kappa$ be u.a.b.-angular and $k=\|\kappa\|_{\infty}$. Then $\kappa$ is extremal if and only if one of the following conditions is true:

(a) $\kappa$ is T.f.n.

(b) $\sup _{x \in \bar{R}}(1 / \pi)\left|\int_{0}^{\pi} \lambda_{x}(\vartheta) e^{-2 i \vartheta} d \vartheta\right|=k$.

From $[\mathbf{F}]$ we know that a complex dilatation $\kappa$ can only be extremal if it is T.f.n. or if there exists a so-called substantial boundary point $x \in \overline{\mathbf{R}}$. In case that $\kappa$ is u.a.b.-angular we will now show that this second case occurs if and only if

$$
\lambda_{x}(\vartheta)=k e^{2 i\left(\vartheta-\vartheta_{0}\right)} \text { a.e. } \vartheta \in(0, \pi) \text { for some point } x \in \overline{\mathbf{R}},
$$

where $\vartheta_{0}$ is a constant.

This shows that in Theorem 1.1 one can replace condition (b) by $\left(b^{\prime}\right)$. In fact, we will show that, if in this theorem condition (b) holds, then this sup is always a max. And, at the same time, we will give a complete proof of a statement which implies Theorem 1.1. Secondly, we will also investigate the question, if the assumption "u.a.b.-angular" can be replaced by simply "angular" in one or both directions of the equivalence. It will turn out that the condition (b) is still sufficient for extremality under this weaker assumption. But, on the other hand, an example of Ortel [O1] shows that there are extremal $\kappa$ 's, not T.f.n., u.a.b.-angular in all points except for one point where $\kappa$ is angular only, where all $\lambda_{x} \equiv 0$, thus showing that condition (b) is not necessary when the uniformity requirement is dropped. A slight modification also shows that we can find a $\kappa$ where condition (b) holds but not condition $\left(b^{\prime}\right)$.

2. Claims and proofs. Let $\kappa \in L_{\infty}(H)$, i.e. a measurable, complex-valued and essentially bounded function in $H$, and let $\|\kappa\|_{\infty}<1$. If the Hamilton condition (1.1) holds, then this sup can be a max, in which case $\kappa$ is T.f.n., or there exists a degenerating Hamilton sequence $\varphi_{n}$, i.e. a sequence $\varphi_{n} \in B(H)$ with $\left\|\varphi_{n}\right\|=1$, $\int_{H} \int \kappa \varphi_{n} d x d y \stackrel{n \rightarrow \infty}{\longrightarrow}\|\kappa\|_{\infty}$ and $\varphi_{n} \stackrel{n \rightarrow \infty}{\longrightarrow} 0$ locally uniformly in $H$ (see [St2]). Let $x \in \overline{\mathbf{R}}$. If there exists a degenerating Hamilton sequence $\varphi_{n}$ such that even $\iint_{H-U}\left|\varphi_{n}\right| d x d y \stackrel{n \rightarrow \infty}{\longrightarrow} 0$ for every neighborhood $U$ of $x$, then we call $x$ a s.b.p. (substantial boundary point) (with respect to $\kappa !)$. This is motivated by the connection of this property with the local dilatation of the induced boundary mapping of $x$ as it is explained in [R]. The following theorem which has been proved in $[\mathbf{F}$ and F-Sa] will be used:

THEOREM 2.1. A complex dilatation $\kappa$ is extremal if and only if $\kappa$ is T.f.n. or there is a s.b.p. (with respect to $\kappa$ ) on $\mathbf{R}$.

Next we will prove

THEOREM 2.2. Let $\kappa$ be angular and $k=\|\kappa\|_{\infty}<1$. The conditions

(1) there is an $x \in \overline{\mathbf{R}}$ with $\lambda_{x}(\vartheta)=k e^{2 i\left(\vartheta-\vartheta_{0}\right)}$ a.e. $\vartheta \in(0, \pi)$;

(2) $\sup _{x \in \mathbf{R}}(1 / \pi)\left|\int_{0}^{\pi} \lambda_{x}(\vartheta) e^{-2 i \vartheta} d \vartheta\right|=k$;

(3) $\kappa$ is extremal and there is a s.b.p. on $\overline{\mathbf{R}}$ are connected as follows:

If $\kappa$ is u.a.b.-angular, then all the conditions are equivalent. If $\kappa$ is angular only, then $(1) \Rightarrow(2)$ and $(2) \Rightarrow(3)$, but it is possible that (2) holds but not (1). Also it is possible that (3) holds but neither (1) nor (2).

We observe that these two theorems imply Theorem 1.1 immediately. 
In what follows we use the notation

$$
D\left(z_{0}, r\right)=\left\{z:\left|z-z_{0}\right|<r\right\} \quad \text { and } \quad D(r)=D(0, r) .
$$

LEMMA 2.1. Let $\kappa \in L^{\infty}(H), k=\|\kappa\|_{\infty}<1$. Let $\kappa$ be angular at a point $x \in$ $\overline{\mathbf{R}}$. Then there is a sequence $\varphi_{n} \in B(H),\left\|\varphi_{n}\right\|=1$ where $\iint_{H-U}\left|\varphi_{n}\right| d x d y \stackrel{n \rightarrow \infty}{\longrightarrow} 0$ for every neighborhood $U$ of $x$ and where

$$
\iint_{H} \varphi_{n} \kappa d x d y \stackrel{n \rightarrow \infty}{\longrightarrow} \frac{1}{\pi} \int_{0}^{\pi} \lambda_{x}(\vartheta) e^{-2 i \vartheta} d \vartheta
$$

PROOF. By conformal invariance we may assume that $x=0$ and put $\lambda(\vartheta)=$ $\lambda_{0}(\vartheta)$. We choose the sequence which is given in [02],

$$
\varphi_{n}(z)=-B_{n} \frac{z^{1 / n-2}}{(z+i)^{1 / n+2}}
$$

where $B_{n}$ is chosen such that $\left\|\varphi_{n}\right\|=1$. One easily concludes that $B_{n}=O(1 / n)$, namely $\varlimsup_{n \rightarrow \infty} n B_{n} \leq 4 / \pi$ and that $\iint_{H-U}\left|\varphi_{n}\right| d x d y \stackrel{n \rightarrow \infty}{\longrightarrow} 0$ for every neighborhood $U$ of $x$. Then we have to show that

$$
\iint_{H} \varphi_{n}\left(r e^{i \vartheta}\right)\left(\kappa\left(r e^{i \vartheta}\right)-\lambda(\vartheta)\right) r d r d \vartheta \stackrel{n \rightarrow \infty}{\longrightarrow} 0 .
$$

Let $\eta>0$. By Egoroff's Theorem there is a measurable set $E_{\eta} \subset(0, \pi)$ of one-dimensional measure $\left|E_{\eta}\right|<\eta$, such that $\kappa\left(r e^{i \vartheta}\right) \stackrel{r \downarrow 0}{\rightarrow} \lambda(\vartheta)$ uniformly for $\vartheta \in$ $(0, \pi)-E_{\eta}$. Let $\varepsilon>0$ and $r(\varepsilon)$ such that

$$
\left|\kappa\left(r e^{i \vartheta}\right)-\lambda(\vartheta)\right|<\varepsilon \quad \text { for } 0<r<r(\varepsilon) \text { and } \vartheta \in(0, \pi) \backslash E_{\eta} .
$$

Obviously, it suffices to show that $\iint_{H \cap D(r(\varepsilon))} \varphi_{n}(\kappa-\lambda) d x d y$ tends to zero. We have

$\left|\iint_{H \cap D(r(\varepsilon))} \varphi_{n}(\kappa-\lambda) d x d y\right| \leq \varepsilon \iint_{H}\left|\varphi_{n}\right| d x d y+2 k \int_{E_{\eta}} \int_{0}^{r(\varepsilon)}\left|\varphi_{n}\left(r e^{i \vartheta}\right)\right| r d r d \vartheta$.

For each $\vartheta \in(0, \pi)$ we have

$$
\int_{0}^{r(\varepsilon)}\left|\varphi_{n}\left(r e^{i \vartheta}\right)\right| r d r \leq B_{n} \int_{0}^{r(\varepsilon)} r^{1 / n-1} d r=n B_{n} r(\varepsilon)^{1 / n}
$$

and hence

$$
\begin{aligned}
\varlimsup_{n \rightarrow \infty}\left|\iint_{H} \varphi_{n}(\kappa-\lambda) d x d y\right| & \leq \varlimsup_{n \rightarrow \infty}\left(\varepsilon|| \varphi_{n} \|+2 k n B_{n} r(\varepsilon)^{1 / n}\left|E_{\eta}\right|\right) \\
& \leq \varepsilon+2 k(4 / \pi) \eta .
\end{aligned}
$$

Since $\varepsilon$ and $\eta$ were arbitrary, (2.2) holds. But, as shown on p. 445 in [O2],

$$
\iint_{H} \varphi_{n}\left(r e^{i \vartheta}\right) \lambda(\vartheta) r d r d \vartheta \stackrel{n \rightarrow \infty}{\longrightarrow} \frac{1}{\pi} \int_{0}^{\pi} \lambda(\vartheta) e^{-2 i \vartheta} d \vartheta
$$

Namely the left side is

$$
\int_{0}^{\pi} \lambda(\vartheta) e^{-2 i \vartheta}\left(\int_{0}^{\infty} \varphi_{n}\left(r e^{i \vartheta}\right) e^{2 i \vartheta} r d r\right) d \vartheta=\int_{0}^{\pi} \lambda(\vartheta) e^{-2 i \vartheta}\left(\int_{0}^{\infty} \varphi_{n}(z) z d z\right) d \vartheta
$$


where the last appearing integral is constant and hence this expression is

$$
\frac{1}{\pi} \int_{0}^{\pi} \lambda(\vartheta) e^{-2 i \vartheta} d \vartheta \cdot \iint_{H} \varphi_{n}(z)\left(\frac{z}{|z|}\right)^{2} d x d y
$$

where the right integral tends to 1 with $n \rightarrow \infty$.

This, together with (2.2), proves (2.1). In particular, this implies

COROLlaRY 2.1. Let $\kappa \in L^{\infty}(H), k=\|\kappa\|_{\infty}<1$. Let $\kappa$ be angular at a point $x \in \overline{\mathbf{R}}$, where $\lambda_{x}(\vartheta)=k e^{2 i\left(\vartheta-\vartheta_{0}\right)}$ for a.a. $\vartheta \in(0, \pi)$ and a constant $\vartheta_{0}$. Then $x$ is a s.b.p.

PROOF. The sequence $\varphi_{n}$ given by Lemma 2.1 satisfies by normality $\varphi_{n} \stackrel{n \rightarrow \infty}{\longrightarrow} 0$ locally uniformly in $H$, hence $e^{2 i \vartheta_{0}} \varphi_{n}$ is a degenerating Hamilton sequence.

Conversely, we have

LEMMA 2.2. Let $\kappa \in L^{\infty}(H), k=\|\kappa\|_{\infty}<1$. Let $\kappa$ be u.a.b.-angular at a s.b.p. $x \in \overline{\mathbf{R}}$. Then

$$
\lambda_{x}(\vartheta)=k e^{2 i\left(\vartheta-\vartheta_{0}\right)} \text { a.e. } \vartheta \in(0, \pi) .
$$

PROOF. By conformal invariance we may again assume that $x=0$ and put $\lambda(\vartheta)=\lambda_{0}(\vartheta)$. By hypothesis there exists a sequence $\varphi_{n} \in B(H),\left\|\varphi_{n}\right\|=1$ with $\iint_{H} \kappa \varphi_{n} d x d y \stackrel{n \rightarrow \infty}{\longrightarrow} k$ and where $\iint_{H-U}\left|\varphi_{n}\right| d x d y \stackrel{n \rightarrow \infty}{\longrightarrow} 0$ for every neighborhood $U$ of 0 . We have to show that

$$
\iint_{H} \varphi_{n}(\kappa-\lambda) d x d y \stackrel{n \rightarrow \infty}{\longrightarrow} 0
$$

because then the result follows from $k=\lim _{n \rightarrow \infty} \iint_{H} \varphi_{n} \lambda d x d y$ and as before

$$
\int_{0}^{\pi} \int_{0}^{\infty} \varphi_{n}\left(r e^{i \vartheta}\right) \lambda(\vartheta) r d r d \vartheta=\frac{1}{\pi} \int_{0}^{\pi} \lambda(\vartheta) e^{-2 i \vartheta} d \vartheta \cdot \iint_{H} \varphi_{n}(z)\left(\frac{z}{|z|}\right)^{2} d x d y
$$

hence

$$
k \leq \lim _{n \rightarrow \infty} \frac{1}{\pi}\left|\int_{0}^{\pi} \lambda(\vartheta) e^{-2 i \vartheta} d \vartheta\right| \cdot|| \varphi_{n} \| \leq \frac{1}{\pi} \int_{0}^{\pi}|\lambda(\vartheta)| d \vartheta \leq k,
$$

i.e., $(1 / \pi)\left|\int_{0}^{\pi} \lambda(\vartheta) e^{-2 i \vartheta} d \vartheta\right|=k$ which means that $\lambda(\vartheta)=k e^{2 i\left(\vartheta-\vartheta_{0}\right)}$. But under our hypothesis, (2.3) has been proved in [02]. For the convenience of the reader we add this proof here. We put

$$
F_{n}(\vartheta)=\int_{0}^{\infty}\left|\varphi_{n}\left(r e^{i \vartheta}\right)\right| r d r
$$

and use the result of [H-I-P], that $F_{n}\left(\right.$ even $\left.\log F_{n}\right)$ is convex for $\vartheta \in(0, \pi)$.

Let $\varepsilon>0$ such that the angularity convergence is uniform for $\vartheta \in(0, \varepsilon) \cup(\pi-\varepsilon, \pi)$. By the convexity above and $\left\|\varphi_{n}\right\|=1$ it follows that $F_{n}(\vartheta) \leq 1 / \varepsilon$ for $\varepsilon \leq \vartheta \leq \pi-\varepsilon$.

Let $\eta>0$ and $E_{\eta} \subset(\varepsilon, \pi-\varepsilon)$ with measure $\left|E_{\eta}\right|<\eta$ such that $\kappa\left(r e^{i \vartheta}\right) \stackrel{r \downarrow 0}{\rightarrow} \lambda(\vartheta)$ uniformly in $(0, \pi) \backslash E_{\eta}$ (Egoroff's theorem and the u.a.b.-angularity!). We choose $r(\eta)>0$ such that

$$
\left|\kappa\left(r e^{i \vartheta}\right)-\lambda(\vartheta)\right|<\eta \quad \text { for } \vartheta \in(0, \pi) \backslash E_{\eta} \text { and } 0<r<r(\eta) .
$$


Then

$$
\begin{aligned}
\left|\iint_{H} \varphi_{n}(\kappa-\lambda) d x d y\right| & \leq \eta\left\|\varphi_{n}\right\|+\int_{E_{\eta}} 2 k F_{n}(\vartheta) d \vartheta+2 k \iint_{H-D(r(\eta))}\left|\varphi_{n}\right| d x d y \\
& \leq \eta+2 k \frac{\eta}{\varepsilon}+2 k \iint_{H-D(r(\eta))}\left|\varphi_{n}\right| d x d y .
\end{aligned}
$$

Hence $\overline{\lim }_{n \rightarrow \infty}\left|\iint_{H} \varphi_{n}(\kappa-\lambda) d x d y\right| \leq \eta+2 k \eta / \varepsilon$ and this holds for all $\eta>0$, hence proving (2.3).

PROOF OF THEOREM 2.2. Let $\kappa$ be angular and $k=\|\kappa\|_{\infty}<1$.

$(1) \Rightarrow(2)$. Trivial.

$(2) \Rightarrow(3)$. We choose a sequence $x_{n} \in \overline{\mathbf{R}}$ with $(1 / \pi)\left|\int_{0}^{\pi} \lambda_{x_{n}}(\vartheta) e^{-2 i \vartheta} d \vartheta\right| \stackrel{n \rightarrow \infty}{\longrightarrow} k$. We then choose a convergent subsequence in $\overline{\mathbf{R}}$ which we call again $x_{n}$ and may assume by conformal invariance that $x_{n} \stackrel{n \rightarrow \infty}{\longrightarrow} 0$. By Lemma 2.1 we can choose for every $x_{n}$ a function $\varphi_{n} \in B(H),\left\|\varphi_{n}\right\|=1$, where

$$
\iint_{H-D\left(x_{n}, 1 / n\right)}\left|\varphi_{n}(z)\right| d x d y<\frac{1}{n}
$$

and $\left|\iint_{H} \kappa \varphi_{n} d x d y-(1 / \pi) \int_{0}^{\pi} \lambda_{x_{n}}(\vartheta) e^{-2 i \vartheta} d \vartheta\right|<1 / n$. Hence, $\left|\iint_{H} \kappa \varphi_{n} d x d y\right|$ $\stackrel{n \rightarrow \infty}{\longrightarrow} k$ and $\iint_{H-U}\left|\varphi_{n}\right| d x d y \stackrel{n \rightarrow \infty}{\longrightarrow} 0$ for every neighborhood $U$ of 0 . Multiplying $\varphi_{n}$ with an appropriate constant $c$ with $|c|=1$, we get a degenerating Hamiltonsequence (by normality it is of course obvious, that also $\varphi_{n} \stackrel{n \rightarrow \infty}{\longrightarrow} 0$ locally uniformly in $H$ ). Hence, $\kappa$ is extremal and 0 a s.b.p.

$(3) \Rightarrow(1)$. If $\kappa$ is u.a.b.-angular at a s.b.p., then this follows by Lemma 2.2 .

To justify the last statement of the theorem, we repeat Ortel's construction [01] of an extremal $\kappa$ which is not T.f.n. This $\kappa$ is u.a.b.-angular at every boundary point except one point, where it is angular only, and all $\lambda_{x}(\vartheta) \equiv 0$.

Choose $k<1$ and $x_{1}>0$. Choose $r_{1}>0$ with $0<x_{1}-r_{1}$. Then consider a sequence $\varphi_{n} \in B(H),\left\|\varphi_{n}\right\|=1$ with $\iint_{H-U}\left|\varphi_{n}\right| d x d y \stackrel{n \rightarrow \infty}{\longrightarrow} 0$ for every neighborhood $U$ of $x_{1}$, e.g. a sequence as in Lemma 2.1. There is a member of this sequence, which we call $\psi_{1}$, and an angle $\vartheta_{1}>0$, such that for $C_{1}=\left\{z \in H:\left|z-x_{1}\right|<r_{1}, \arg z>\vartheta_{1}\right\}$

$$
\iint_{C_{1}}\left|\psi_{1}\right| d x d y \geq \frac{1}{2}
$$

Then we choose $x_{2}>0$, with $x_{2}<x_{1}-r_{1}$ and $r_{2}>0$ such that $D\left(x_{2}, r_{2}\right) \cap\{z \in$ $\left.H: \arg z<\vartheta_{1}\right\}=\varnothing$. In the corresponding sequence $\varphi_{n} \in B(H)$ associated to $x_{2}$, we determine a function, $\psi_{2}$, and an angle $\vartheta_{2}$, where

$$
\iint_{C_{2}}\left|\psi_{2}\right| d x d y \geq \frac{2}{3}
$$

for $C_{2}=\left\{z \in H:\left|z-x_{2}\right|<r_{2}, \arg z_{2}>\vartheta_{2}\right\}$. Clearly, we get sequences $x_{n} \downarrow 0$, $\vartheta_{n} \downarrow 0, C_{n}$ and $\psi_{n}$ with

The example

$$
\iint_{C_{n}}\left|\psi_{n}\right| d x d y \geq \frac{n}{n+1}
$$

$$
\kappa(z)= \begin{cases}k \bar{\psi}_{n}(z) /\left|\psi_{n}(z)\right|, & z \in C_{n}, n=1,2, \ldots \\ 0, & z \in H-\bigcup_{n=1}^{\infty} C_{n}\end{cases}
$$


has the desired properties, since

$$
\iint_{H} \kappa \psi_{n} d x d y=k \iint_{C_{n}}\left|\psi_{n}\right| d x d y+k \sum_{i \neq n} \iint_{C_{i}} \psi_{n} \bar{\psi}_{i} /\left|\psi_{i}\right| d x d y
$$

and

$$
\sum_{i \neq n} \iint_{C_{i}}\left|\psi_{n}\right| d x d y \leq 1 /(n+1),
$$

hence $\lim _{n \rightarrow \infty} \iint_{H} \kappa \psi_{n} d x d y=k$. So $\kappa$ is extremal and 0 a s.b.p. A slight modification now shows that also (2) in Theorem 2.2 can be made to hold without (1) holding. In every disk $D\left(x_{n}, r_{n}\right)$ we choose a smaller disk $D_{n}$ with center $x_{n}$ such that $D_{n} \cap C_{n}=\varnothing$. Then we define

$$
\kappa(z)= \begin{cases}k \bar{\psi}_{n}(z) /\left|\psi_{n}(z)\right|, & z \in C_{n}, n=1,2, \ldots, \\ k n /(n+1) e^{2 i \arg \left(z-x_{n}\right)}, & z \in D_{n} \cap H, n=1,2, \ldots, \\ 0, & z \in H-\bigcup_{n=1}^{\infty} C_{n}-\bigcup_{n=1}^{\infty} D_{n} .\end{cases}
$$

Evidently $(1 / \pi) \int_{0}^{\pi} \lambda_{x_{n}}(\vartheta) e^{-2 i \vartheta} d \vartheta=k n /(n+1) \stackrel{n \rightarrow \infty}{\longrightarrow} k$, but $\lambda_{x}(\vartheta) \equiv 0$ for all $x \in \overline{\mathbf{R}} \backslash\left\{x_{1}, x_{2}, \ldots\right\}$. We have no point where the sup in (2) is assumed and again $\left|\iint_{H} \kappa \psi_{n} d x d y\right| \geq k \iint_{C_{n}}\left|\psi_{n}\right| d x d y-k \iint_{H-C_{n}}\left|\psi_{n}\right| d x d y \geq k n /(n+1)-k /(n+1)$. So, if $c$ is chosen appropriately with $|c|=1$, then $\lim _{n \rightarrow \infty} \iint_{H} c \psi_{n} \kappa d x d y=k$. Hence $\kappa$ is extremal and 0 a s.b.p.

FINAL REMARK. The content of the treated problem can also be stated as follows. We map $H$ conformally onto an infinite horizontal strip. The complex dilatation satisfies $\left(\mathrm{b}^{\prime}\right)$ (following Theorem 1.1) at the preimage of $-\infty$ iff the induced complex dilatation tends to $k e^{2 i \vartheta_{0}}$ along a.e. horizontal line as one moves to $-\infty$. Hence, this point is a s.b.p. for a u.a.b.-angular $\kappa$ iff these limit values are the same as in the case of an affine stretch.

\section{REFERENCES}

[F] R. Fehlmann, Über extremale quasikonforme Abbildungen, Comment. Math. Helv. 56 (1981), 558-580.

[F-Sa] R. Fehlmann and K. Sakan, On the set of substantial boundary points for extremal quasiconformal mappings, Complex Variables, Theory and Appl. 6 (1986), 323-335.

[H] R. S. Hamilton, Extremal quasiconformal mappings with prescribed boundary values, Trans. Amer. Math. Soc. 138 (1969), 399-406.

[H-I-P] G. H. Hardy, A. E. Ingham and G. Polya, Theorems concerning mean values of analytic functions, Proc. Roy. Soc. Ser. A 113 (1927), 542-569.

[O1] M. Ortel, Integral means and the theorem of Hamilton, Reich and Strebel, Complex Analysis, Joensuu 1978 (Proc. Colloq. Complex Analysis, Joensuu, Finland), Ilpo Laine, Olli Lehto, and Tuomas Sorvali, eds., Lecture Notes in Math., vol. 747, Springer-Verlag, Berlin, Heidelberg and New York, 1979, pp. 301-308.

[02] _ Extremal quasiconformal mappings with angular complex dilatation, Indiana Univ. Math. J. 31 (1982), 435-447.

[R] E. Reich, On the relation between local and global properties of boundary values for extremal quasiconformal mappings, Discontinuous Groups and Riemann Surfaces, Ann. of Math. Stud., no. 79, Princeton Univ. Press, 1974, pp. 391-407.

[R-St] E. Reich and K. Strebel, Extremal quasiconformal mappings with given boundary values, Contributions to Analysis, A Collection of Papers Dedicated to Lipman Bers, Academic Press, New York, 1974, pp. 375-391. 
[St1] K. Strebel, Zur Frage der Eindeutigkeit extremaler quasikonformer Abbildungen des Einheitskreises. II, Comment. Math. Helv. 39 (1964), 77-89.

[St2] _ On the existence of extremal Teichmüller mappings, J. Analyse Math. 30 (1976), 464480 .

Nokia Research Center, P. O. BoX 780, 00101 Helsinki, Finland 